4 Cecilia Rocchi ${ }^{\S}$, Camille Louvat ${ }^{1 \S}$, Adriana Miele ${ }^{2}$, Julien Batisse ${ }^{3}$, Christophe Guillon ${ }^{1}$, Lionel

5 Ballut ${ }^{1}$, Daniela Lener ${ }^{4}$, Matteo Negroni ${ }^{4}$, Marc Ruff ${ }^{3}$, Patrice Gouet ${ }^{1}$ and Francesca Fiorini ${ }^{*}$.

$6{ }^{1}$ Molecular Microbiology and Structural Biochemistry, MMSB-IBCP, UMR 5086 CNRS University 7 of Lyon, 7 passage du Vercors, 69367, Lyon Cedex 07, France.

8

\section{The HIV-1 Integrase C-Terminal domain induces TAR RNA structural changes promoting Tat binding.}

2 Institute of Analytical Science
69100 Villeurbanne, France.

${ }^{3}$ Chromatin Stability and DNA Mobility, Department of Integrated Structural Biology, IGBMC, CNRS UMR 7104 - Inserm U 158 University of Strasbourg, 1 rue Laurent Fries, 67404, Illkirch 12 Cedex, France.

${ }^{4}$ RNA architecture and reactivity, IBMC, CNRS UPR 9002 University of Strasbourg, 2, Allée Konrad Roentgen, 67084 Strasbourg Cedex, France.

$\S$ : equal contribution

*To whom correspondence should be addressed: francesca.fiorini@ibcp.fr

\section{ABSTRACT}

Recent evidence indicated that HIV-1 Integrase (IN) binds genomic viral RNA (gRNA) playing a critical role in viral particle morphogenesis and gRNA stability in host cells. Combining biophysical

22 and biochemical approaches we show that the C-terminal flexible 18-residues tail of IN acts as a 3 sensor of the peculiar apical structure of trans-activation response element RNA (TAR), directly 4 interacting with its hexaloop. We highlighted how the whole IN C-terminal domain, once bound to 25 TAR, can change its structure assisting the binding of Tat, the HIV trans-activator protein, which finally displaces IN from TAR. Our results are consistent with the emerging role of IN in early stage 7 of proviral transcription and suggest new steps of HIV-1 life cycle that can be considered as 28 therapeutic targets. 


\section{INTRODUCTION}

Protein-nucleic acid interactions can occur through different types of protein binding domains and are responsible for a variety of essential molecular and cellular mechanisms and their regulation. This binding diversity has been well described by recent RNA interactome screenings revealing that the term RNA-Binding Domain (RBD) is no longer synonymous with a well-structured domain, but also with intrinsically disordered region (IDR) with non-canonical RNAbinding properties ${ }^{1} 23$. Moreover, an increasing level of complexity has been documented for certain transcription factors that are able to bind both DNA and RNA through separated structured or unstructured regions, resulting in a complex pattern of specific and non-specific interactions ${ }^{4}$ 5 . This protein moonlighting is particularly true for RNA viruses which possess a relatively short genome encoding only for a small amount of proteins that must ensure many multiple functions during viral replication ${ }^{6} 7$.

A good example of moonlighting is represented by Human Immunodeficiency Virus type 1 (HIV1) integrase (IN) which can bind both DNA and RNA ${ }^{8,9}$. As for all retroviruses, IN catalyzes the integration of viral cDNA (vDNA), produced by retro-transcription of genomic RNA (gRNA), into the host genome. Multimeric IN binds the vDNA ends forming the intasome complex able to catalyze the processing of 3'-end dinucleotides. After activation, the 3' extremities are then used by the intasome to attack the host DNA in order to integrate the provirus (reviewed in ${ }^{9}$ ). A step essential for HIV-1 productive infection ${ }^{10}$. Retroviral integrases are modular proteins that contain three structured domains: the N-terminal domain (NTD), the catalytic core domain (CCD) and the C-terminal domain (CTD), connected by unstructured regions. All three domains show proteinprotein and protein-DNA interaction properties and are essential for enzymatic activity (reviewed in ${ }^{9}$ and ${ }^{11}$ ). The CCD harbors the essential catalytic triad D, D, E that coordinates two $\mathrm{Mg}^{2+}$ cofactors and folds similarly to nucleotidyl-transferases and nucleases ${ }^{12}$. The NTD is involved in enzyme multimerization and catalytic activity and shows a HHCC motif coordinating a $\mathrm{Zn}^{2+}$ ion ${ }^{13}$ 14 15. The CTD is also involved in DNA interaction, multmerization, and possesses a SH3-like fold followed by a flexible 18-residues tail (CT) ${ }^{16} 1718$ 19. The SH3-like domain of IN is the minimal DNA binding site. In HIV-1 life cycle, this hub domain mediates the interaction with RT, with cellular nuclear import complex TRN-SR2; and with histone 4 tail, likely anchoring the intasome to the chromatin and therefore promoting an efficient integration 20212223 . The mutational study of flexible CT revealed a moderate implication in IN enzymatic activity ${ }^{21}{ }^{24}$, but significant effect on the incorporation of IN in virions and on HIV-1 infectivity ${ }^{21}$. However the exact function of $\mathrm{CT}$ region remains largely unknown. 
As mentioned before, recent works revealed that in HIV-1, IN is also an RBP with an essential role in virion morphogenesis, related to its ability to bind gRNA 82526 . In fact, IN interacts with specific sites within gRNA ensuring the correct localization of viral RNP inside the capsid (reviewed in ${ }^{27}$ ). Aberrant virions are obtained when IN-gRNA interaction is abolished, highlighting the importance of the proper formation of IN-containing RNPs for HIV-1 infection $\left({ }^{8,25,28}\right)$. In addition, the IN-gRNA interaction is also dictating the fate of the gRNA within the host cell in the early steps of infection. Indeed, when virions are defective for IN-gRNA interactions, viral infection is blocked at early stages of reverse transcription, due to a rapid degradation of gRNA in host cells 25,28 . If the RBD within IN has not yet been structurally identified, most of the Lysine residues interacting with RNA are located within the CTD and overlap with those subjected to posttranslational modification 8293031 . Importantly, Lysine 273 , belonging to the flexible CT, seems to be the only Lysine dedicated to RNA interaction to be essential for viral infectivity ${ }^{8}$.

Recently, a new role has been proposed for HIV-1 IN during proviral transcription at early times after integration. In fact, after strand transfer, the IN remains bound to DNA and directly plays a role in proviral transcription, depending on its post-translational modifications of specific residues within the CTD ${ }^{32}$.

HIV-1 provirus is transcribed by the cellular RNA polymerase II (Pol II) which pauses shortly after initiation of transcription, due to the presence of negative elongation factors as well as nucleosomes downstream the transcription start site ${ }^{33} 34$. HIV-1 removes this block by encoding a transcriptional trans-activator Tat protein, which binds the nascent transcript on a structured RNA sequence named TAR (trans-activation response element RNA) using a non-canonical RBD. This allows the recruitment of the human super elongation complex (SEC) 35363738 . In particular, Tat binds to $\mathrm{p}$-TEFb, a complex composed of CDK9 kinase and its regulatory partner the cyclin T1 (CycT1), and consequently drives SEC to TAR RNA. This complex triggers a cascade of phosphorylation of several transcription factors, which activate Pol II and recruit positive chromatin remodelers. Moreover, processivity of Pol II is also enhanced by pTEFb-mediated phosphorylation of its C-terminal domain ${ }^{39}$ (reviewed in ${ }^{40}$ ). Unfortunately, molecular details about the interplay between IN and this cellular transcription initiation machinery are largely unknown so far.

Crosslinking-immuno-precipitation sequencing (CLIP-seq) experiments have identified the TAR RNA sequence as a major binding site of HIV-1 IN on the gRNA ${ }^{8}$. This observation, together with the recent finding of HIV-1 IN involvement in proviral transcription ${ }^{32}$, prompted us to study the interaction of IN with TAR RNA and its interplay with the Tat protein. Our results revealed that despite the apparent lack of structural specificity of IN in vitro, the CT flexible tail discriminates for 
the proper TAR apical stem-loop. We described the consequences of the IN binding on the structure of TAR and on the subsequent Tat/TAR interaction, proposing a working model which foresees a possible involvement of IN in proviral transcription elongation before the arrival of Tat.

\section{RESULTS}

\section{IN binds TAR RNA with no apparent structural specificity.}

We first addressed whether full-length HIV-1 IN was able to specifically bind TAR RNA. One hindering aspect of this study is the well-known low solubility of HIV-1 IN as well as its flexibility between N-terminal (NTD), C-terminal (CTD) and catalytic core (CCD) domains that for long time frustrated structural studies (Fig. 1a ${ }^{41}$ ). The poor solubility in vitro is usually overcome by mutations of hydrophobic residues, however resulting in a replication-defective virus with mislocalized viral RNP phenotype analogous to that observed in IN mutants defective for the INgRNA interaction ${ }^{19} 8,25,28$. We have chosen to express recombinant wild-type N-Terminal Flagtagged IN-FL in eukaryotic expression system as previously described ( ${ }^{42}$ Supplementary Fig. 1a, left panel) and called it IN-FLm (1-288 amino acids [aa]). Mass spectrometry analysis of this protein revealed post-translational modification at several residues: Serine 24 was phosphorylated and Lysine 46, 173, 211 and 273 residues were acetylated. The protein produced in mammalian cells was shown to have an increased solubility as compared to that produced in $E$. coli and also an enhanced enzymatic activity in vitro ${ }^{42}$. However, after purification, we kept the protein in solution after buffer optimization. In this study we used an electrophoretic mobility shift assay (EMSA) in order to assess qualitatively the binding of IN to a synthetic TAR RNA, whose secondary structure is depicted in Fig. 1b, to a weakly structured $\mathrm{RNA}_{(30)}$-mer (Supplementary Fig. 1b, left panel) and to an unstructured $A_{(50)}$-mer RNA (Fig. 1c). We have incubated radiolabeled RNA with increasing concentrations of purified IN-FLm. A band shift was observed with TAR RNA upon gel electrophoresis under nearly physiological salt concentration, reflecting the formation of an IN:TAR complex (Fig. 1c). IN-FLm also bound weakly-structured $\mathrm{RNA}_{(30)}$-mer (Supplementary Fig. 1b, right panel), while it had a markedly reduced affinity for unstructured $A G_{(50)}$-mer RNA (Fig. 1c). IN-FL produced in prokaryotic expression system (Supplementary Fig. 1a, right panel) is also able to bind TAR (Supplementary Fig. 1c).

As RNA-interacting lysine residues are contained within the C-terminal domain of $I N^{8}$, we focused on this domain. We expressed and purified the whole C-Terminal Domain (IN-CTD; aa 
222-288). We first determined whether IN-CTD was able to interact with structurally distinct viral genomic RNA elements of similar nucleotide length derived from HIV-15' UTR. In addition to TAR, we synthesized three other RNA hairpins: the polyadenylation (polyA) signal, the dimerization initiation sequence (DIS) and the major splice-donor (SD) together with $\Psi$ packaging element (Psi) (Fig. 1d, top panel). IN-CTD bound all those RNAs with no measurable difference (Fig. 1d, bottom panel), suggesting that IN can bind structured RNA through its C-terminal domain.

Subsequently, we wanted to address whether the C-terminal flexible tail (CT) spanning the last 18 residues of CTD affected the RNA binding properties of IN. We expressed the CTD without its terminal tail (IN-CTD- $\Delta$ CT; aa 220-270, Fig. 1a) and used a chemically synthesized IN-CT peptide (aa 270-288; Fig. 1a). The boundaries between CTD and CT were defined according to sequence alignment and previous structural studies ${ }^{16} 43,4445$. The IN-CT is not conserved among lentiviruses (Supplementary Fig. 2a), however, multiple alignment of IN-CT from human HIV-1 subtypes and simian viruses (Fig. 1e) showed a $58 \%$ of sequence identity and $87 \%$ of similarity (equivalent residues considering physical-chemical properties).

EMSA assays did not shown apparent differences between the affinities of IN-CTD and IN-CTD- $\triangle C$ T for TAR under physiological salt conditions (Supplementary Fig. 2b). To measure the rate constants of the IN-CTD and IN-CTD- $\triangle C T$ interactions with TAR, we used Bio-layer interferometry (BLI)= A 3' biotinylated TAR RNA, was immobilized on a streptavidin-coated biosensor to serve as a bait molecule (Fig. 1f, top panel). Subsequently, the interactions of IN subdomains with the TAR RNA were monitored in real time and the resulting sensorgrams are shown in Supplementary Fig. 2c. Fitted data resulted in $K_{D}$ values of 0.77 and $0.32 \mu \mathrm{M}$ for INCTD- $\triangle \mathrm{CT}$ and IN-CTD, respectively (Fig. 1f, bottom panel). Thus, the presence of the 18 aa Cterminal tail only slightly affects the RNA binding affinity of IN-CTD.

\section{The C-terminal Tail senses the TAR RNA shape.}

It has been shown that the presence of the bulge and the loop in TAR RNA, rather than its sequence, is critical for IN binding, as the deletion of one or both markedly decreased IN binding affinity ${ }^{8}$. We wondered whether the shape of the peculiar apical stem-loop of TAR (Fig. 2a) could also be important for IN binding. Therefore, we mutated the 4- nucleotides (nt) stem between the bulge and the loop to change its length, as shortening or lengthening the 4 nt-stem is likely to tighten or loosen the TAR major groove by bringing the bulge and the hexa-loop closer or further, respectively. We produced TAR with 1 bp longer stem (TAR-LS) and two with progressively 
shorter stem TARs (TAR-SS and TAR-VSS) (Fig. 2a). The EMSA assay showed that IN-FLm was able to bind TAR-LS similarly to the wild-type TAR (Supplementary Fig. 3a, lines A and B). On the contrary, shortening of the stem reduced IN-FLm affinity for TAR (Supplementary Fig. 3a, lines $\mathrm{C}$ to $\mathrm{E}$ ). Furthermore, we assessed the binding behavior of IN-CTD and INCTD- $\triangle \mathrm{CT}$ to TAR mutants by EMSA (Fig. 2b and 2c). As observed for the full-length protein, the affinity of IN-CTD for TAR mutants decreased as the length of the stem was reduced (Fig. 2b, lines A to D and Fig. 2c). Surprisingly, the binding ability of IN-CTD- $\triangle C T$ was not affected by the shortening of the TAR 4nt-stem (Fig. 2b, lines A' to D', and Fig. 2c), suggesting that IN C-terminal tail senses the shape of the TAR RNA stem in vitro.

As a control, we studied the interaction of Tat with TAR, the cognate interacting protein. We used a chemically synthesized full-length Tat protein (1-102 aa) to assess the binding with TAR mutants by EMSA assay. Similarly to IN-CTD and consistently with other in vitro and ex vivo information 464748 , the binding ability of Tat decreased with stem shortening (Supplementary Fig. 3b).

Overall, our results suggest that IN-CT interacts with the apical stem-loop of TAR, possibly with its major groove. This is consistent with the CLIP-seq data, which had identified the TAR hexaloop as a major binding site for IN and with a recently published structural model of IN-CTD$\triangle \mathrm{CT}$ bound with TAR ${ }^{8} 50$.

\section{IN-CTD deeply affect the structure of TAR favoring Tat interaction.}

In order to extend the binding analysis at the molecular level, the IN-TAR interaction was probed by footprinting techniques. 5' radiolabeled TAR, alone or complexed to protein, was subjected to partial digestion using RNase T1 that cleaves 3' to an unpaired guanine. The results shown in Fig. 3a indicated that G34 and G36 of TAR were protected in the presence of IN-CTD (Fig. 3a, lane 6), instead the binding of IN-CT and IN-CTD- $\triangle$ CT did not protect these nucleotides from nuclease digestion (Fig. 3a, lanes 4 and 5). This suggested that the CT region is interacting with G34 and G36 nucleotides located in the TAR hexaloop and its junction with the 4-nt stem when CT is part of the whole domain (Fig. 3a, lane 6). Surprisingly, we observed prominent cleavage following the residues at positions C41, U38, G32 and, to a lesser extent, G28, induced by the binding of IN-CTD- $\triangle \mathrm{CT}$ and IN-CTD even in the absence of T1 nuclease (Supplementary Fig. 4a, left panel). This reflected the presence of drastic structural constraints on the apical stemloop of TAR upon the binding of either IN-CTD- $\triangle$ CT or IN-CTD, which leads to a spontaneous 
192

193

194

195

196

197

198

199

200

201

202

203

204

205

206

207

208

209

210

211

212

213

214

215

216

217

218

219

220

221

222

223

mechanical breakage. Any nuclease contamination has been observed in protein solution (Supplementary Fig. 4a, right panel). Altogether, these digestion patterns showing the protection of G34 and G36 by IN-CTD, but not IN-CTD- $\triangle C T$, as well as the evidence of the structural deformation of TAR induced by the binding of both fragments, indicate that: (i) IN-CTD binds to the TAR apical stem-loop; (ii) IN-CTD modifies the structure of the RNA; (iii) the CT exerts a specific role in the interaction of IN-CTD with the TAR hexaloop and its junction with the stem. The fact that CT binds TAR only in the IN-CTD context, could reflect the necessity for a distortion of TAR by the IN-CTD- $\triangle C T$ moiety to allow for the correct binding of CT to the hexaloop. Therefore the role of Tat seems to be that of coating TAR which resulted in a prevention of nuclease digestion (Fig. 3a, lane 7).

In order to understand whether the structural changes of TAR induced by IN were affecting the binding affinity of Tat, we performed displacement experiments by BLI. 3' biotinylated TAR was previously immobilized to streptavidin-coated biosensor, then IN association to TAR was monitored as described before. Afterwards, the sensor was absorbed in solutions containing various concentrations of Tat (Fig. 3b). We calculated the apparent $\mathrm{K}_{\mathrm{D}}$ by measuring the $\Delta$ Wavelength Shift between the minimum wavelength after Tat injection (around $400 \mathrm{~s}$ ) and the maximum wavelength reach at $600 \mathrm{~s}$ (Supplementary Fig. 4c), and we plotted the values against the corresponding Tat concentrations (Fig. 3c). The $K_{D}$ of Tat binding to TAR was about 37.5 and 75 times lower in presence of IN-CTD- $\triangle C$ CT and IN-CTD respectively (Fig. 3c) compared to the $\mathrm{K}_{D}$ of Tat measured in the same conditions for the naked RNA (Fig. 3d and Supplementary Fig. 4d). Moreover, the binding kinetics of Tat on IN/TAR complex is consistent with a cooperative interaction (Fig. 3c). Notably, the IN-CTD:TAR complex can accommodate more Tat than IN-CTD$\triangle \mathrm{CT}: T A R$, as indicated by the higher $\mathrm{B}_{\operatorname{Max}}$ (Fig. 3c). To exclude that these results derived from a direct interaction between IN-CTD and Tat, we performed a pulldown assay which did not show protein-protein interaction in these conditions (Supplementary Fig. 4e).

\section{Tat competes with IN-CTD and displaces it from TAR.}

To investigate the mechanism involved in Tat binding to IN:TAR complexes, we performed a dose-dependent competition EMSA (Fig. 4a), in the presence of $10 \mathrm{~K}_{\mathrm{D}}$ IN/TAR ratio. We could confirm that Tat binds to TAR in a dose-response manner (Fig. 4a, lanes 13-17). Interestingly, we observed a dose-dependent interference between Tat and IN-CTD for TAR binding at intermediate (Fig. 4a, lanes 4 and 5), but not at high concentrations of Tat (Fig. 4a, lane 6). Furthermore, no 
224 interference was observed at any concentration for IN-CTD- $\Delta C T$ (Fig. 4a, lanes 9 to 12), 225 confirming that the mechanism was dependent on the presence of the CT tail. Since the migration 226 pattern of the EMSA cannot discriminate between IN-bound and Tat-bound RNAs, we assessed 227 whether the presence of Tat would modify the binding of IN to 3' biotinylated TAR by pull-down 228 assays (Fig. 4b). IN-CTD and IN-CTD- $\triangle$ CT were efficiently co-precipitated (Fig. 4b, lanes 1 and 2295 , respectively), whereas the addition of increasing concentrations of Tat serially decreased their 230 binding to TAR (Fig. 4b, lanes 2 to 4 and 6 to 8, respectively). Control experiments showed that 231 Tat also interacted with TAR and was not precipitated when biotinylated TAR was absent (Fig. 232 4b, lanes 9 and 10). Altogether these results underline a competition of Tat and IN for TAR binding. 233 The fact that very small differences were detected between IN-CTD and IN-CTD- $\triangle$ CT during TAR234 pulldown assay (Fig. 4b), while they were observed in EMSA (Fig. 4a) is likely due to the higher 235 sensitivity offered by the latter, in which nanomolar concentrations of proteins and RNA were used.

\section{DISCUSSION}

The C-terminal tail of HIV-1 IN ensures several functions essential for infectivity. A comprehensive understanding of its involvement in the various steps of the infectious cycle is still lacking due to a lack of structural information and to the pleiotropic effects caused by its mutations 5121 . Here, we found that this intrinsically disordered region of IN acts as a specific sensor for the peculiar structure of the apical stem-loop of TAR RNA and is directly interacting with its apical hexaloop. We probed IN/TAR interaction by EMSA, nuclease digestion and bio layer interferometry. Data analysis suggested that IN-CTD modified TAR conformation, leading to an enhanced binding of Tat, especially when CT region is present. Moreover, we put forth evidence for an interplay between Tat, IN and TAR, where Tat is competing with IN-CTD for TAR binding and destabilizes preformed IN-CTD:TAR complex.

Mutations of TAR aimed at altering the peculiar structure of the apical portion decreased the relative affinity of IN-CTD compared to IN-CTD- $\triangle \mathrm{CT}$, suggesting a role of $\mathrm{CT}$ in the recognition of this portion of TAR structure (Fig. 2). Importantly, full-length IN presented the same trend of sensitivity for TAR structural mutants (Supplementary Fig. 3a). The presence of CT does not seem to be associated with a selective specificity of IN for TAR in vitro, as IN-CTD could bind efficiently to other structured RNAs regardless of the presence of CT. In particular, IN-CTD interacts with polyA, DIS, and SD/ $\Psi$ RNA elements of HIV-1 5'UTR (Fig. 1d). Consistently, the $\mathrm{K}_{D}$ measured for IN interaction with all these gRNA elements has been shown to span a narrow range 
256

257

258

259

260

261

262

263

264

265

266

267

268

269

270

271

272

273

274

275

276

277

278

279

280

281

282

283

284

285

286

287

288

of values ${ }^{8}$. The better affinity previously observed for the full-length IN ${ }^{8}$ compared to IN-CTD measured in this work (Fig. 1f and Supplementary Fig. 2b and c) could be due to the presence of additional RNA binding sites within the NTD and CCD domains of full-length IN ${ }^{8}$ and/or to differences in analytical techniques with respect to those employed here. Thus, the CT region can recognize a TAR RNA with the proper apical stem-loop conformation among possible structural defective TAR conformers, but it is not involved in the discrimination between viral structured RNA regions. Interestingly, this behavior of IN-CT reminds that of $\mathrm{p} 6$, the C-terminal domain of HIV-1 $\operatorname{Pr} 55^{\text {Gag }} 52$. Pr55 ${ }^{\mathrm{Gag} \Delta \mathrm{p} 6}$ mutant, deleted of the p6 domain, showed no RNA binding specificity compared to the full lengths Pr55 Gag, suggesting that the presence of this region is required for the specific binding of Pr55 ${ }^{\text {Gag }}$ to DIS RNA within the 5'UTR of HIV-1 genome ${ }^{52}$.

Previous biochemical and structural studies have demonstrated that unstructured Tat arginine-rich motif (ARM) penetrates in the TAR major groove made by stem-bulge-stem-loop secondary structures and mostly interacts with the U-rich bulge and nearby double-stranded regions (Supplementary Fig. 5a; 38535455 ). Despite the absence in the CT of a motif comparable to the Arginine stretch of Tat, our results suggest that the unstructured IN-CT region also binds the TAR major groove, but through the interaction with G34 and, to a lesser extent G36, of the hexaloop (Fig. 3a). This could explain the lower affinity of IN-CTD for TAR mutants (Fig. 2): likely because the altered position of G34 and G36. Interestingly, in a recent report, the IN-CTD:TAR complex has been modeled on the base of the IN-CTD:INI $1_{183-304}$ structure, based on the fact that the same 6 residues were engaged for the binding of IN-CTD to INI 1 and TAR ( ${ }^{8,50}$ Supplementary Fig. 5b). In this model, IN-CTD binds the minor groove of the 4-nt apical stem of TAR. Noteworthy, the last C-terminal modeled residue (D270), immediately preceding the CT tail, is oriented towards the major groove of TAR, which is consistent with our hypothesis ( ${ }^{50}$ Supplementary Fig. 5b).

The interaction of IN with gRNA has been shown to be critical for the proper localization of VRNP inside the protective capsid lattice ${ }^{8,25,26}$. In this context, the TAR-selection activity is exerted by IN-CT, while in the Pr160 Gag-Pol precursor, could be an additional mechanism to that of the Pr55 ${ }^{\text {Gag }}$ protein interaction with the packaging signals (reviewed in ${ }^{56}$ ) in order to selectively recruit and encaspsidate the HIV-1 gRNA in the viral core.

Our observations show how the interplay between IN, Tat and TAR RNA takes place: first IN-CTD binds TAR through its C-terminal tail by contacting the apical stem-loop and in particular the hexaloop. This interaction modifies the structure of TAR (Fig. 3a) favoring Tat binding (Fig. 3b and 3c), which finally displaces IN from the TAR RNA (Fig. 4). Noteworthy, the competition of Tat ARM with IN for TAR binding has also been reported elsewhere ${ }^{8}$. Our data are also fully 
consistent with recent evidences of the implication of $\mathrm{IN}$ in the transcription of the provirus at early times after integration and in a Tat-independent manner ${ }^{32}$. The authors found that mutation of four lysine residues within the IN-CTD dramatically reduced proviral transcription since all of them are involved in the binding of IN to the viral RNA ${ }^{8,26}$.

Taken together, these in vitro observations suggest a working model in which IN would ensure the very first stages of proviral transcription (Fig. 5): IN-CTD, through to its CT tail, selectively binds to the nascent TAR transcript. This binding modifies the structure of TAR, facilitating its interaction with Tat. Therein Tat displaces IN and allows the subsequent transactivation of provirus transcription (Fig. 5). This process is dynamic and might also be modulated by post-translational modifications of IN, interaction with cellular partners and/or chromatin remodeling processes.

Interestingly, the structure of the HIV-1 intasome during the strand transfer process, revealed that the inner IN tetrameric core, which contacts both the viral and host DNA, is surrounded by twelve other subunits which display a considerable flexibility ${ }^{57}$ and might be available for other functions. Unfortunately, the structure of IN after strand transfer is not yet available and the prediction of the presence of CTDs available for transcription is not possible so far.

\section{METHODS}

\section{Protein expression and purification}

Construction of plasmid pET15b, encoding N-terminal 6XHis tagged IN-CTD (aa 220-270) and INCTD- $\triangle C T$ (aa 220-288) were previously reported ${ }^{44}$. IN-FL gene from pNL4-3 were cloned in pPROEX-HTa vector in frame with 6 XHis tag at $\mathrm{N}$-terminus. All proteins were expressed in Escherichia coli BL21(DE3) Rosetta/pLysS strain (Novagen). Cells were grown in LB medium supplemented with $10 \%(\mathrm{w} / \mathrm{v})$ glucose and protein expression was induced at an optical density at $600 \mathrm{~nm}\left(\mathrm{OD}_{600}\right)$ of 0.6 with $1 \mathrm{mM}$ IPTG (isopropyl- $\beta$-d-thiogalactopyranoside). Cells were incubated overnight at $18^{\circ} \mathrm{C}$ under continuous shaking, then pelleted. The cell pellets were resuspended in lysis buffer composed by $50 \mathrm{mM}$ Tris- $\mathrm{HCl}$ pH 8, $1 \mathrm{M} \mathrm{NaCl}, 20 \mathrm{mM}$ imidazole, 0.1 mM EDTA, $2 \mathrm{mM} \beta$-mercaptoethanol and 10\% (w/v) glycerol. Exclusively for bacterial lysis the buffer was freshly supplemented with $2 \mathrm{M}$ urea, $2 \mathrm{mM}$ of Adenosine triphosphate (ATP), $5 \mathrm{mM}$ CHAPS (3-[(3-cholamidopropyl)dimethylammonio]-1-propanesulfonate) and 1 tablet of Protease Inhibitor Cocktail (ROCHE, cOmplete ${ }^{\mathrm{TM}}$ ). The preparation was sonicated for 120 s on ice, then the 
321 resulting lysate was subjected to centrifugation at $11.000 \mathrm{~g}$ for $1 \mathrm{~h}$. The recovered supernatant was 322 then applied to a HisTrap ${ }^{\mathrm{TM}}$ Fast Flow Crude column (Cytiva) and purified by AKTA pure system 323 (Cytiva). The sample was first abundantly washed with lysis buffer containing $100 \mathrm{mM}$ imidazole 324 and $2 \mathrm{M} \mathrm{NaCl}$, then the protein was eluted using a gradient up to $500 \mathrm{mM}$ imidazole in 10 column 325 volumes. A second step of purification was carried out using a Superdex 75 10/300 GL column 326 (Cytiva) by an isocratic elution carried out with storing buffer (50 mM HEPES pH 7.5, 1 M NaCl, 5 $327 \mathrm{mM} \beta$-mercaptoethanol, and 5\% glycerol).

328 FlagIN-FL (IN-FLm) was expressed in Baby Hamster Kidney suspension cells (BHK21-C13-2P, 329 Sigma-Aldrich) using a vaccinia virus expression system as previously described ${ }^{42}$.

330 IN-CT peptide (YGKQMAGDDCVASRQDED) and 101-residue long Tat protein of primary isolate 331133 of HIV-1 were chemically synthetized ${ }^{58} 59$. This Tat has been biochemically characterized and 332 its full biological activity was previously validated ${ }^{60}$.

\section{In vitro RNA synthesis, purification, and radiolabeling.}

334 We produced several RNAs as listed in Table 1 by using partially double-stranded templates formed by hybridization of T7 promoter-containing DNA oligonucleotides listed in Table 2 and following the protocol detailed in ${ }^{61}$. Templates for polyA, DIS and SD/Psi RNAs were produced by PCR using pNL4-3 plasmid as template and T7 promoter-containing primers. RNA was transcribed by kit MEGAshortscript ${ }^{\mathrm{TM}} \mathrm{T7}$ (Thermo Fisher Scientific) following the manufacturer instructions, de-phosphorylated using Alkaline Phosphatase (New England Biolabs) and purified by phenol:chloroform:isoamyl alcohol (25:24:1) extraction and ethanol precipitation. The 3' biotinylated TAR and RNA 30-mer (Table 1) were chemically synthesized (Sigma Aldrich). RNA 342 (50 pmol) was radiolabelled at 5' end with by using 10 units of T4 polynucleotide kinase (New 343 England Biolabs) mixed to $3 \mu \mathrm{l}$ of $\mathrm{\gamma}^{32} \mathrm{P}$-ATP $(3000 \mathrm{Ci} / \mathrm{mmol} 10 \mathrm{mCi} / \mathrm{ml}$, Perkin Elmer) for $1 \mathrm{~h}$ at $37^{\circ} \mathrm{C}$ then purified on denaturing $10 \%(\mathrm{w} / \mathrm{v})$ polyacrylamide gel $(29: 1)$ as previously described (Fiorini et al 2012). Before use in binding and structural studies, RNA was heated in refolding buffer (20 mM HEPES pH 7.5, 0.2 M NaCl, $2 \mathrm{mM} \mathrm{MgCl}_{2}, 2 \mathrm{mM} \mathrm{DTT}$ ) for $3 \mathrm{~min}$ at $95^{\circ} \mathrm{C}$, followed by 40 min of slow controlled cooling to room temperature, and finally placed on ice.

\section{Electrophoretic Mobility Shift Assays (EMSA)}

349 The electrophoretic mobility shift assays (EMSA) were performed as described in ${ }^{62}$. Samples 350 were prepared by mixing a radiolabelled RNA with increasing concentrations of proteins, as 351 indicated, in a buffer containing $20 \mathrm{mM}$ MES pH 6.0, $150 \mathrm{mM} \mathrm{NaCl}, 2 \mathrm{mM}$ DTT, $2 \mathrm{mM} \mathrm{MgCl}_{2}$, $3520.2 \mu \mathrm{g} \mathrm{BSA}$ and $8 \%(\mathrm{v} / \mathrm{v})$ PEG8000. The samples were incubated at $37^{\circ} \mathrm{C}$ for 30 min before being 
353

354

355

356

357

358

359

360

361

362

363

364

365

366

367

368

369

370

371

372

373

374

375

376

377

378

379

380

381

382

383

384

resolved by native $6 \%$ polyacrylamide (19:1) gel electrophoresis in 0.5x TAE (Tris acetate EDTA) buffer. Results were analysed by phosphorimaging using ImageQuant software. IN-FLm was incubated for $2 \mathrm{~h}$ at $4^{\circ} \mathrm{C}$ with the RNA substrate. For dose-dependent competition assay showed in Fig. $4 \mathrm{a}$, we used in all the samples a constant saturating IN-to-TAR concentrations with an INto-TAR ratio per sample exceeding about ten times their respective $K_{D}$. Then, we added increasing concentrations of Tat.

\section{RNA Structural probing}

Enzymatic treatments were performed in $10 \mu \mathrm{l}$ of reaction mix containing $0.5 \mathrm{pmol}$ of 5 ' radiolabeled RNA, $0.2 \mu \mathrm{g}$ of yeast tRNA, $1 \times$ Structure buffer (Thermo Fisher Scientific) and 0.01 $\mathrm{U}$ of RNase T1 (Thermo Fisher Scientific). Incubation was done at $37^{\circ} \mathrm{C}$ for $5 \mathrm{~min}$. Reactions were stopped by addition of $40 \mu \mathrm{l}$ of quenching buffer composed by $10 \mathrm{mM} \mathrm{HEPES} \mathrm{pH} \mathrm{7.5,} 1 \mathrm{mM}$ EDTA and $3 \%$ SDS. Partial alkaline hydrolysis was performed as follows: $10 \mu$ of reaction mix containing $0.2 \mathrm{pmol}$ of RNA, $1 \mu \mathrm{g}$ of yeast tRNA, $1 \times$ Alkaline Hydrolysis buffer, were incubated at $95^{\circ} \mathrm{C}$ for 12 min then quenched wih $2 x$ denaturing loading buffer and placed on ice and. For RNA/protein complexes, 0.5 pmol RNA was previously incubated with $36 \mathrm{pmol}$ of protein at $37^{\circ} \mathrm{C}$ for $30 \mathrm{~min}$ then treated with RNase T1. After quenching, samples were phenol extracted and ethanol precipitated. After recovery from precipitation, all samples were run on a $15 \%$ sequencing polyacrylamide gel in $0.5 \times$ TBE. (Tris Borate EDTA). Results were analysed by phosphorimaging.

\section{Pulldown assay}

The pulldown assays were conducted as described before ${ }^{61}$. Briefly, proteins were mixed in binding buffer (40 mM HEPES pH 7.5, $2.5 \mathrm{mM} \mathrm{MgCl}_{2}, 2 \mathrm{mM} \beta$-mercaptoethanol and 5\% glycerol) adjusting the final $\mathrm{NaCl}$ concentration to $150 \mathrm{mM}$. Samples were complemented or not with 600 pmol of 3' end-biotinylated TAR (Table 1) in a final volume of $30 \mu \mathrm{l}$, and incubated $30 \mathrm{~min}$ at 37 ${ }^{\circ} \mathrm{C}$. To the mix were added $8 \mu$ of magnetic streptavidin beads (Dynabeads MyOne, Thermo Fischer Scientific) and further incubated for $1 \mathrm{~h}$ at $4^{\circ} \mathrm{C}$ in gentle rotation. The resin was washed three times with $500 \mu \mathrm{l} \mathrm{BB}-200$ (40 mM HEPES pH 7.5, $200 \mathrm{mM} \mathrm{NaCl}, 2.5 \mathrm{mM} \mathrm{MgCl}, 2 \mathrm{mM} \beta$ mercaptoethanol and 10\% glycerol) on ice and proteins were eluted with SDS loading buffer and analysed on polyacrylamide 16\% (37.5:1) SDS-PAGE. For Histidine pulldown assay, magnetic streptavidin resin was replaced with HisPur ${ }^{\text {TM }}$ Ni-NTA Magnetic Beads and protein elution was done with $0.5 \mathrm{M}$-Imidazole containing buffer.

\section{Bio-Layer interferometry}


385

386

387

388

389

390

391

392

393

394

395

396

397

398

399

400

401

402

403

404

405

406

407

408

409

410

411

412

413

414

415

For Bio-Layer Interferometry (BLI) analysis we used the BLItz platform (FortéBio). For high throughput experiments such as shown in Fig. 1 and Supplementary Fig. 2e we used the Octet RED96e System (FortéBio). All Measurements were performed in assay buffer composed of 50 $\mathrm{mM}$ Hepes $\mathrm{pH}$ 7.5, $200 \mathrm{mM} \mathrm{NaCl}, 2 \mathrm{mM} \beta$-mercaptoethanol, $5 \%$ glycerol, $2 \mathrm{mM} \mathrm{MgCl}, 1 \mu \mathrm{M}$ $\mathrm{ZnSO}_{4}, 0.5 \mathrm{M}$ BSA. For BLItz platform we used Streptavidin (SA) Biosensors (ForteBio) and for Octet RED96e the Streptavidin (SAX) Biosensors (FortéBio) that were hydrated for $10 \mathrm{~min}$ in assay buffer then plunged in a solution containing $1 \mu \mathrm{M} 3^{\prime}$ end-biotinylated TAR RNA in assay buffer with 1x RNAase inhibitor (RNA Secure, Invitrogen) and $0.1 \mu \mathrm{M}$ BSA for the RNA loading step. A wash was performed after RNA loading. In BLItz experiment the association of the protein to RNA was monitored in real-time for $200 \mathrm{~s}$ : the hydrated biosensor tip was placed in a $1.5 \mathrm{ml}$ black assay tube containing $200 \mu$ of protein solution in assay buffer as indicated. Afterward, a dissociation step or a second association with Tat, was performed for $200 \mathrm{~s}$. Biosensors were discarded after each measurement. All kinetic assays showed in Fig. 1 and Supplementary Fig. 2 e were performed using Octet RED96e system and carried out using black 96-well plates and samples were diluted in freshly prepared assay buffer and incubated at $37^{\circ} \mathrm{C}$ with an orbital shake. Association and dissociation steps were as in the BLItz experiments. Each time reference sensors and negative control sensors were included. Sensorgrams were exported and data analysis was performed with Kaleida Graph software (Synergy Software).

\section{Acknowledgements}

This work was supported by the French Agency for Research on AIDS and Viral Hepatitis (ECTZ72240-1 to F.F., M.R. and M.N.; PhD fellowship for C.R.). We acknowledge: the support and the use of resources of the French Infrastructure for Integrated Structural Biology FRISBI ANR-10-INBS-05 and of Instruct-ERIC; Sylvie Ricard-Blum and the Interaction platform of the UMR 5246 ICBMS for the experiments on the Octet RED96e; the Protein Science Facility (PSF) of SFR Biosciences (UAR3444/CNRS, US8/Inserm, ENS de Lyon, UCBL) especially Virginie Gueguen-Chaignon and Eric Desis for their assistance, and Frédéric Galisson for the help with radioactive sources. We are grateful to the Viral DNA Integration and Chromatin Dynamics Network (DyNAVir) for fruitful discussions and to Anne-Catherine Dock-Bregeon for critical reading of the manuscript. We acknowledge Andrea Cimarelli for the pNL4-3 plasmid donation. 


\section{Author Contributions}

417 C.R., C.L., and F.F. conceived and designed experiments. C.R., C.L., A.E.M., C.G., L.B. D.L. and

418 F.F. performed the experiments and/or analyzed data. J.B. and M.R. prepared protein from 419 mammalian expression system; C.R., C.L., A.E.M., C.G., L.B. D.L, M.N., M.R., P.G and. F.F. 420 discussed the results; F.F. wrote the paper. All authors read and edited the manuscript.

\section{Competing Interests statement}

423 The authors declare no competing interests.

\section{References}

426

427

428

429

430

431

432

433

434

435

436

437

438

439

440

441

442

443

444

445

446

447

448

449

450

451

452

453

454

455

1. Castello, A. et al. Insights into RNA biology from an atlas of mammalian mRNA-binding proteins. Cell 149, 1393-406 (2012).

2. Beckmann, B.M., Castello, A. \& Medenbach, J. The expanding universe of ribonucleoproteins: of novel RNA-binding proteins and unconventional interactions. Pflugers Arch 468, 1029-40 (2016).

3. Hentze, M.W., Castello, A., Schwarzl, T. \& Preiss, T. A brave new world of RNA-binding proteins. Nat Rev Mol Cell Biol 19, 327-341 (2018).

4. Cassiday, L.A. \& Maher, L.J., 3rd. Having it both ways: transcription factors that bind DNA and RNA. Nucleic Acids Res 30, 4118-26 (2002).

5. Brodsky, S. et al. Intrinsically Disordered Regions Direct Transcription Factor In Vivo Binding Specificity. Mol Cell 79, 459-471 e4 (2020).

6. Garcia-Moreno, M., Jarvelin, A.I. \& Castello, A. Unconventional RNA-binding proteins step into the virus-host battlefront. Wiley Interdiscip Rev RNA 9, e1498 (2018).

7. Basu, S. \& Bahadur, R.P. A structural perspective of RNA recognition by intrinsically disordered proteins. Cell Mol Life Sci 73, 4075-84 (2016).

8. Kessl, J.J. et al. HIV-1 Integrase Binds the Viral RNA Genome and Is Essential during Virion Morphogenesis. Cell 166, 1257-1268 e12 (2016).

9. Maertens, G.N., Engelman, A.N. \& Cherepanov, P. Structure and function of retroviral integrase. Nat Rev Microbiol (2021).

10. Sakai, H. et al. Integration is essential for efficient gene expression of human immunodeficiency virus type 1. J Virol 67, 1169-74 (1993).

11. Esposito, D. \& Craigie, R. HIV integrase structure and function. Adv Virus Res 52, 319-33 (1999).

12. Dyda, F. et al. Crystal structure of the catalytic domain of HIV-1 integrase: similarity to other polynucleotidyl transferases. Science 266, 1981-6 (1994).

13. Zheng, R., Jenkins, T.M. \& Craigie, R. Zinc folds the N-terminal domain of HIV-1 integrase, promotes multimerization, and enhances catalytic activity. Proc Natl Acad Sci U S A 93, 13659-64 (1996).

14. Cai, M. et al. Solution structure of the N-terminal zinc binding domain of HIV-1 integrase. Nat Struct Biol 4, 567-77 (1997).

15. Lee, S.P., Xiao, J., Knutson, J.R., Lewis, M.S. \& Han, M.K. Zn2+ promotes the self-association of human immunodeficiency virus type-1 integrase in vitro. Biochemistry 36, 173-80 (1997). 
456

457

458

459

460

461

462

463

464

465

466

467

468

469

470

471

472

473

474

475

476

477

478

479

480

481

482

483

484

485

486

487

488

489

490

491

492

493

494

495

496

497

498

499

500

501

502

16. Eijkelenboom, A.P. et al. The DNA-binding domain of HIV-1 integrase has an SH3-like fold. Nat Struct Biol 2, 807-10 (1995).

17. Woerner, A.M. \& Marcus-Sekura, C.J. Characterization of a DNA binding domain in the Cterminus of HIV-1 integrase by deletion mutagenesis. Nucleic Acids Res 21, 3507-11 (1993).

18. Engelman, A., Hickman, A.B. \& Craigie, R. The core and carboxyl-terminal domains of the integrase protein of human immunodeficiency virus type 1 each contribute to nonspecific DNA binding. J Virol 68, 5911-7 (1994).

19. Jenkins, T.M., Engelman, A., Ghirlando, R. \& Craigie, R. A soluble active mutant of HIV-1 integrase: involvement of both the core and carboxyl-terminal domains in multimerization. $J$ Biol Chem 271, 7712-8 (1996).

20. Lutzke, R.A., Vink, C. \& Plasterk, R.H. Characterization of the minimal DNA-binding domain of the HIV integrase protein. Nucleic Acids Res 22, 4125-31 (1994).

21. Dar, M.J. et al. Biochemical and virological analysis of the 18-residue C-terminal tail of HIV-1 integrase. Retrovirology 6, 94 (2009).

22. De Houwer, S. et al. Identification of residues in the C-terminal domain of HIV-1 integrase that mediate binding to the transportin-SR2 protein. J Biol Chem 287, 34059-68 (2012).

23. Mauro, E. et al. Human $\mathrm{H} 4$ tail stimulates HIV-1 integration through binding to the carboxyterminal domain of integrase. Nucleic Acids Res 47, 3607-3618 (2019).

24. Vink, C., Oude Groeneger, A.M. \& Plasterk, R.H. Identification of the catalytic and DNA-binding region of the human immunodeficiency virus type I integrase protein. Nucleic Acids Res 21, 141925 (1993).

25. Madison, M.K. et al. Allosteric HIV-1 Integrase Inhibitors Lead to Premature Degradation of the Viral RNA Genome and Integrase in Target Cells. J Virol 91(2017).

26. Elliott, J.L. et al. Integrase-RNA interactions underscore the critical role of integrase in HIV-1 virion morphogenesis. Elife $\mathbf{9}(2020)$.

27. Elliott, J.L. \& Kutluay, S.B. Going beyond Integration: The Emerging Role of HIV-1 Integrase in Virion Morphogenesis. Viruses 12(2020).

28. Fontana, J. et al. Distribution and Redistribution of HIV-1 Nucleocapsid Protein in Immature, Mature, and Integrase-Inhibited Virions: a Role for Integrase in Maturation. J Virol 89, 9765-80 (2015).

29. Cereseto, A. et al. Acetylation of HIV-1 integrase by p300 regulates viral integration. EMBO J 24, 3070-81 (2005).

30. Manganaro, L. et al. Concerted action of cellular JNK and Pin1 restricts HIV-1 genome integration to activated CD4+ T lymphocytes. Nat Med 16, 329-33 (2010).

31. Zamborlini, A. et al. Impairment of human immunodeficiency virus type-1 integrase SUMOylation correlates with an early replication defect. J Biol Chem 286, 21013-22 (2011).

32. Winans, S. \& Goff, S.P. Mutations altering acetylated residues in the CTD of HIV-1 integrase cause defects in proviral transcription at early times after integration of viral DNA. PLoS Pathog 16, e1009147 (2020).

33. Jonkers, I. \& Lis, J.T. Getting up to speed with transcription elongation by RNA polymerase II. Nat Rev Mol Cell Biol 16, 167-77 (2015).

34. Mousseau, G. \& Valente, S.T. Role of Host Factors on the Regulation of Tat-Mediated HIV-1 Transcription. Curr Pharm Des 23, 4079-4090 (2017).

35. Schulze-Gahmen, U. \& Hurley, J.H. Structural mechanism for HIV-1 TAR loop recognition by Tat and the super elongation complex. Proc Natl Acad Sci U S A 115, 12973-12978 (2018).

36. Sobhian, B. et al. HIV-1 Tat assembles a multifunctional transcription elongation complex and stably associates with the 7SK snRNP. Mol Cell 38, 439-51 (2010). 
503

504

505

506

507

508

509

510

511

512

513

514

515

516

517

518

519

520

521

522

523

524

525

526

527

528

529

530

531

532

533

534

535

536

537

538

539

540

541

542

543

544

545

546

547

548

37. He, N. et al. HIV-1 Tat and host AFF4 recruit two transcription elongation factors into a bifunctional complex for coordinated activation of HIV-1 transcription. Mol Cell 38, 428-38 (2010).

38. Pham, V.V. et al. HIV-1 Tat interactions with cellular 7SK and viral TAR RNAs identifies dual structural mimicry. Nat Commun 9, 4266 (2018).

39. Wei, P., Garber, M.E., Fang, S.M., Fischer, W.H. \& Jones, K.A. A novel CDK9-associated C-type cyclin interacts directly with HIV-1 Tat and mediates its high-affinity, loop-specific binding to TAR RNA. Cell 92, 451-62 (1998).

40. Ne, E., Palstra, R.J. \& Mahmoudi, T. Transcription: Insights From the HIV-1 Promoter. Int Rev Cell Mol Biol 335, 191-243 (2018).

41. Craigie, R. The molecular biology of HIV integrase. Future Virol 7, 679-686 (2012).

42. Levy, N. et al. Production of unstable proteins through the formation of stable core complexes. Nat Commun 7, 10932 (2016).

43. Eijkelenboom, A.P. et al. Refined solution structure of the C-terminal DNA-binding domain of human immunovirus-1 integrase. Proteins 36, 556-64 (1999).

44. Kanja, M. et al. NKNK: a New Essential Motif in the C-Terminal Domain of HIV-1 Group M Integrases. J Viro/ 94(2020).

45. Lodi, P.J. et al. Solution structure of the DNA binding domain of HIV-1 integrase. Biochemistry 34, 9826-33 (1995).

46. Berkhout, B. \& Jeang, K.T. Detailed mutational analysis of TAR RNA: critical spacing between the bulge and loop recognition domains. Nucleic Acids Res 19, 6169-76 (1991).

47. Churcher, M.J. et al. High affinity binding of TAR RNA by the human immunodeficiency virus type- 1 tat protein requires base-pairs in the RNA stem and amino acid residues flanking the basic region. J Mol Biol 230, 90-110 (1993).

48. Roy, S., Delling, U., Chen, C.H., Rosen, C.A. \& Sonenberg, N. A bulge structure in HIV-1 TAR RNA is required for Tat binding and Tat-mediated trans-activation. Genes Dev 4, 1365-73 (1990).

49. Delling, U. et al. Conserved nucleotides in the TAR RNA stem of human immunodeficiency virus type 1 are critical for Tat binding and trans activation: model for TAR RNA tertiary structure. $J$ Virol 66, 3018-25 (1992).

50. Dixit, U. et al. INI1/SMARCB1 Rpt1 domain mimics TAR RNA in binding to integrase to facilitate HIV-1 replication. Nat Commun 12, 2743 (2021).

51. Lu, R., Ghory, H.Z. \& Engelman, A. Genetic analyses of conserved residues in the carboxylterminal domain of human immunodeficiency virus type 1 integrase. J Virol 79, 10356-68 (2005).

52. Dubois, N. et al. The C-terminal p6 domain of the HIV-1 Pr55(Gag) precursor is required for specific binding to the genomic RNA. RNA Biol 15, 923-936 (2018).

53. Puglisi, J.D., Tan, R., Calnan, B.J., Frankel, A.D. \& Williamson, J.R. Conformation of the TAR RNAarginine complex by NMR spectroscopy. Science 257, 76-80 (1992).

54. Weeks, K.M., Ampe, C., Schultz, S.C., Steitz, T.A. \& Crothers, D.M. Fragments of the HIV-1 Tat protein specifically bind TAR RNA. Science 249, 1281-5 (1990).

55. Cordingley, M.G. et al. Sequence-specific interaction of Tat protein and Tat peptides with the transactivation-responsive sequence element of human immunodeficiency virus type 1 in vitro. Proc Natl Acad Sci U S A 87, 8985-9 (1990).

56. Comas-Garcia, M., Davis, S.R. \& Rein, A. On the Selective Packaging of Genomic RNA by HIV-1. Viruses 8(2016).

57. Passos, D.O. et al. Cryo-EM structures and atomic model of the HIV-1 strand transfer complex intasome. Science 355, 89-92 (2017). 
549

550

551

552

553

554

555

556

557

558

559

560

561

562

563

564

565

566

567

568

569

570

571

572

573

574

575

576

577

578

579

580

581

582

583

584

585

586

587

58. Guillon, C., Stankovic, K., Ataman-Onal, Y., Biron, F. \& Verrier, B. Evidence for CTL-mediated selection of Tat and Rev mutants after the onset of the asymptomatic period during HIV type 1 infection. AIDS Res Hum Retroviruses 22, 1283-92 (2006).

59. Mayol, K., Munier, S., Beck, A., Verrier, B. \& Guillon, C. Design and characterization of an HIV-1 Tat mutant: inactivation of viral and cellular functions but not antigenicity. Vaccine 25, 6047-60 (2007).

60. Foucault, M. et al. UV and X-ray structural studies of a 101-residue long Tat protein from a HIV-1 primary isolate and of its mutated, detoxified, vaccine candidate. Proteins 78, 1441-56 (2010).

61. Fiorini, F., Bonneau, F. \& Le Hir, H. Biochemical characterization of the RNA helicase UPF1 involved in nonsense-mediated mRNA decay. Methods Enzymol 511, 255-74 (2012).

62. Fiorini, F., Boudvillain, M. \& Le Hir, H. Tight intramolecular regulation of the human Upf1 helicase by its N- and C-terminal domains. Nucleic Acids Res 41, 2404-15 (2013).

63. Gruber, A.R., Lorenz, R., Bernhart, S.H., Neubock, R. \& Hofacker, I.L. The Vienna RNA websuite. Nucleic Acids Res 36, W70-4 (2008).

64. Lorenz, R. et al. ViennaRNA Package 2.0. Algorithms Mol Biol 6, 26 (2011).

65. Robert, X. \& Gouet, P. Deciphering key features in protein structures with the new ENDscript server. Nucleic Acids Res 42, W320-4 (2014).

66. Muller-Esparza, H., Osorio-Valeriano, M., Steube, N., Thanbichler, M. \& Randau, L. Bio-Layer Interferometry Analysis of the Target Binding Activity of CRISPR-Cas Effector Complexes. Front Mol Biosci 7, 98 (2020).

Figure 1: IN binds to structured RNA. a Schematic diagram showing the domain organization of IN: the N-terminal domain (NTD), the catalytic core domain (CCD) and the Cterminal domain (CTD) are indicated respectively in green, orange and blue rectangles. Recombinant protein versions used in our study are represented by grey lines: IN full-length from mammalian expression system (IN-FLm); IN full-length from E. coli (IN-FL); IN-CTD (residues 222 to 288); IN-CTD- $\triangle \mathrm{CT}$ (222 to 270); IN-CT (270 to 288). Sites of phosphorylation (P) and acetylation (Ac) are shown in red. b Structural model of TAR RNA obtained with RNA Fold WebServer ${ }^{63} 64$ predicting a minimum free energy of $-29.60 \mathrm{kcal} / \mathrm{mol}$. c Representative native $6 \%$ polyacrylamide gel illustrating the Electrophoretic mobility shift assay (EMSA) showing the interaction of IN-FLm with TAR RNA or an unstructured RNA 50-mer ( $\mathrm{AG}_{(50)}$-mer) labelled with ${ }^{32} \mathrm{P}$ (black star). The RNA substrates $(50 \mathrm{nM})$ were incubated with various concentrations of IN-FLm or without protein (TAR RNA: 0; 100, 200, 400 nM of IN-FLm; AG $_{(50)}$-mer RNA: 0 or 400 nM of INFLm). d Models of secondary structures of four RNA elements belonging to 5 ' untranslated region of the HIV-1 RNA genome: TAR, Poly-A, DIS and SD/Psi (upper panel). EMSA assay showing the binding of IN-CTD to these RNA elements (lower panel). Increasing amounts of IN-CTD (0; 100; 200; $400 \mathrm{nM}$ ) were incubated with 5'-end radiolabeled RNA (50nM). e Sequence alignment of the C-terminal extremity of IN-CTD from HIV-1 subtypes and simian viruses. All amino acid sequences 
588

589

590

591

592

593

594

595

596

597

598

599

600

601

602

603

604

605

606

607

608

609

610

611

612

613

614

615

616

617

618

619

were obtained from the HIV database compendium (http://www.hiv.lanl.gov/) and aligned using Clustal Omega (https://www.ebi.ac.uk/Tools/msa/clustalo/) in order to have a consensus sequence for each viral subtype. Consensus sequences from subtypes $A 1, B, C, G, N, O$ and from GOR and CPZ, where aligned and analyzed by ESPript 3.0 Web server ${ }^{65}$. Secondary structure elements from IN-CTD- $\triangle C$ T structure (PDB code: 5TC2) were presented on top of the alignment (helices with squiggles and strands with arrows). Red shading indicates sequence identity and boxes indicate sequence similarity, according to physical-chemical properties. $f$ top: Schematic representation of the Bio-Layer interferometry (BLI) experiment showing the binding of IN (grey ellipses) to 5'-biotinylated TAR RNA immobilized on streptavidin-coated biosensor; bottom: graph showing the wavelength shifts recorded at $200 \mathrm{~s}$ after the start of the protein/RNA binding were plotted against the corresponding IN-CTD- $\triangle C T$ (blue line, squares) and IN-CTD (red line, circles) concentrations, in order to calculate the respective equilibrium dissociation constant $\left(K_{D}\right)$ values. Data points were fitted to the equation: $Y=B_{\max }{ }^{*} X /(K d+X)+N S^{*} X$ where $B_{\max }$ is the maximum wavelength shift and NS the slope of the non-linear component as described in ${ }^{66}$. The coefficients of determination $\left(R^{2}\right)$ and equilibrium dissociation constant $\left(K_{D}\right)$ values obtained are indicated in the graph for each IN fragment. Binding assays were performed in duplicate. Error bars indicate the Standard Error of the Mean. 
620 5'-end radiolabeled TAR (34-mer) was incubated in the presence or absence of protein for $30 \mathrm{~min}$ 621 at $37^{\circ} \mathrm{C}$ prior to RNAse $\mathrm{T} 1$ treatment, as described in experimental procedures. RNA fragments 622 were separated on a 15\% denaturing polyacrylamide sequencing gel. Bands corresponding to 623 certain T1 cleavage (at $\mathrm{G}$ bases) products are identified as position markers. Probing gel lanes 624 are as follows: (M) Ladder of two RNA transcripts of 33 and 20 nucleotides in length (lane 1); $625\left(\mathrm{AC}(40) \mathrm{AH}\right.$ ) alkaline ladder of $\mathrm{AC}_{(40)}$-mer RNA (lane 2); TAR native (lane3); TAR RNA complexes 626 with IN-CT (lane 4); IN-CTD- $\triangle$ CT (lane 5); IN-CTD (lane 6) and Tat (lane 7). Digestion patterns 627 were mapped on TAR secondary structure depicted on the right of the gel: circles identify 628 nucleotides protected from RNAse T1 digestion and arrows mark the RNA cleavage sites. Grey 629 arrows indicated nonspecific hydrolysis. The dimensions of arrows are proportional to the intensity 630 of the band. b Real-time measurements of protein-RNA interaction obtained using Interferometry 631 assays (BLItz ${ }^{\circledR}$ System instrument- FortéBio). The 3' biotinylated TAR (Bt-TAR) was first loaded 632 on the streptavidin-coated biosensor for $100 \mathrm{~s}$ (Bt-association) then the unbound RNA was 633 washed for $50 \mathrm{~s}$ (baseline). The sensor was absorbed in a solution containing about $90 \mu \mathrm{M}$ of IN 634 protein for $200 \mathrm{~s}$ than incubated with different concentrations of Tat $(0.2,0.5,1$ and $2 \mu \mathrm{M})$ for 200 635 s. c Graph showing the wavelength shifts recorded $200 \mathrm{~s}$ after Tat addition (at $600 \mathrm{~s}$ of kinetics) 636 normalized on the minimum wavelength shifts measured at the moment of Tat addition (400 s), 637 as a function of the corresponding Tat concentrations. Data points were fitted with Hill equation: $638 y=B \max x^{n} / K_{D}+\left(x^{n}\right)$. d BLI experiment to measure the affinity of Tat for immobilized Bt-TAR RNA: 639 graph showing the wavelength shifts recorded after $200 \mathrm{~s}$ after the start of the protein/RNA binding 640 were plotted against the corresponding Tat concentrations. Data points were fitted with Michaelis641 Menten equation and $R^{2}$ and $K_{D}$ values were shown in the graphs inset.

642

643

Figure 4. Tat competes with IN-CTD for TAR binding. a EMSA assay showing a dose644 dependent competition assay of Tat on IN-CTD/TAR or IN-CTD- $\triangle C T / T A R$ complexes.

645 Radiolabeled TAR (50 nM) was incubated with 16 fold molar excess of IN-CTD (lanes 2-6) or IN646 CTD- $\triangle C T$ (lanes 8-12) with or without increasing concentration of Tat $(25,50,100,200 \mathrm{nM}$ ). Tat $647 v s$ IN-CTD is shown in lanes 3-6 and IN-CTD- $\triangle$ CT in lanes 9-12. Control binding of Tat on TAR 648 was also performed using Tat alone (lanes 13-17). b Protein co-precipitation with $3^{\prime}$ end649 biotinylated TAR (Bt-TAR). IN-CTD (lanes 1-4) or IN-CTD- $\triangle$ CT (lanes 5 and 8) were mixed with 650 increasing amount of Tat (1, 2, $3 \mu \mathrm{g} /$ sample; lanes 2, 3, 4 and 6, 7, 8) and incubated in a buffer 651 containing $200 \mathrm{mM} \mathrm{NaCl}$ before co-precipitation. Tat was incubated with Bt-TAR alone (lane 9) or 
652 TAR-free beads as a control for unspecific binding (lane 10). Input (20\% of total) and pull-down 653 fractions were analyzed by $15 \%$ SDS-PAGE followed by Coomassie blue staining.

654

655

Figure 5. Working Model proposed for the interplay of IN-CTD, Tat and TAR during the 656 early state of HIV-1 proviral transcription. Once the integration has taken place, IN interacts with 657 nascent TAR transcript through its CTD, directly interacting with the major groove and the 658 hexaloop, thanks to its C-terminal tail. The binding induces TAR conformational changes, which 659 promote the interaction of Tat with its substrate. Afterwards, Tat displaces IN-CTD from TAR and 660 recruits the SEC complex in order to boost Pol II transcription.

661

662

663

664

665 


\section{Figure 1}

a

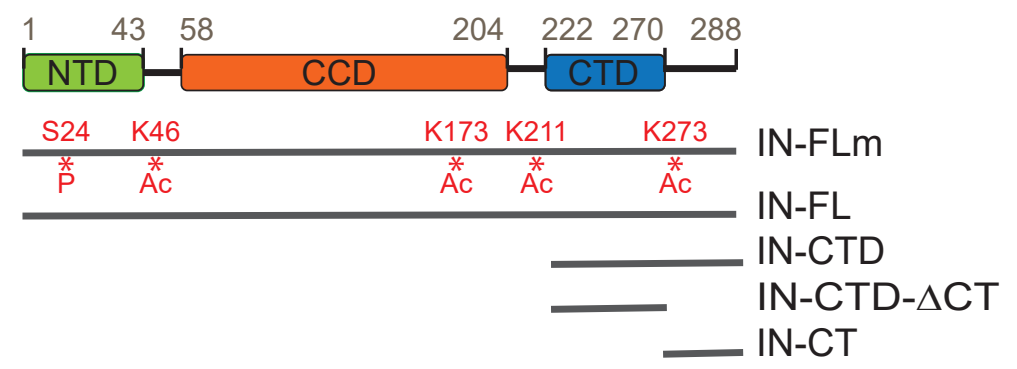

b

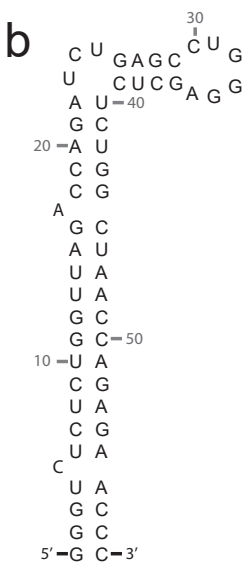

TAR

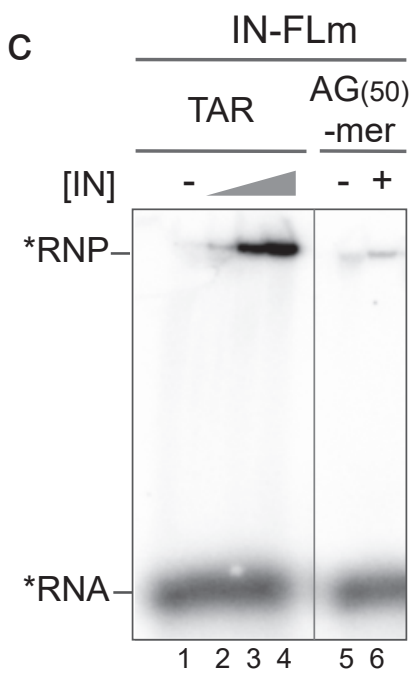

e

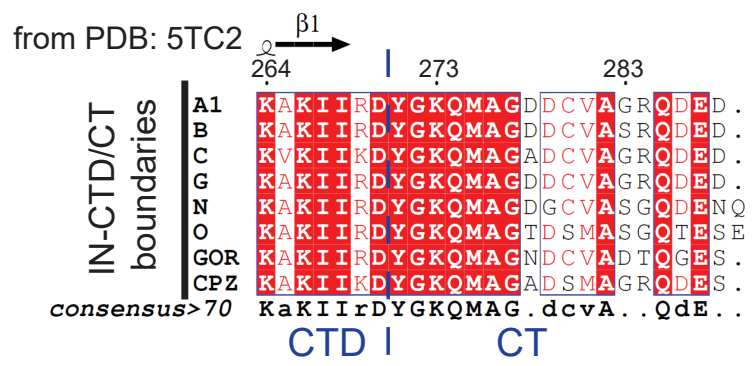

d
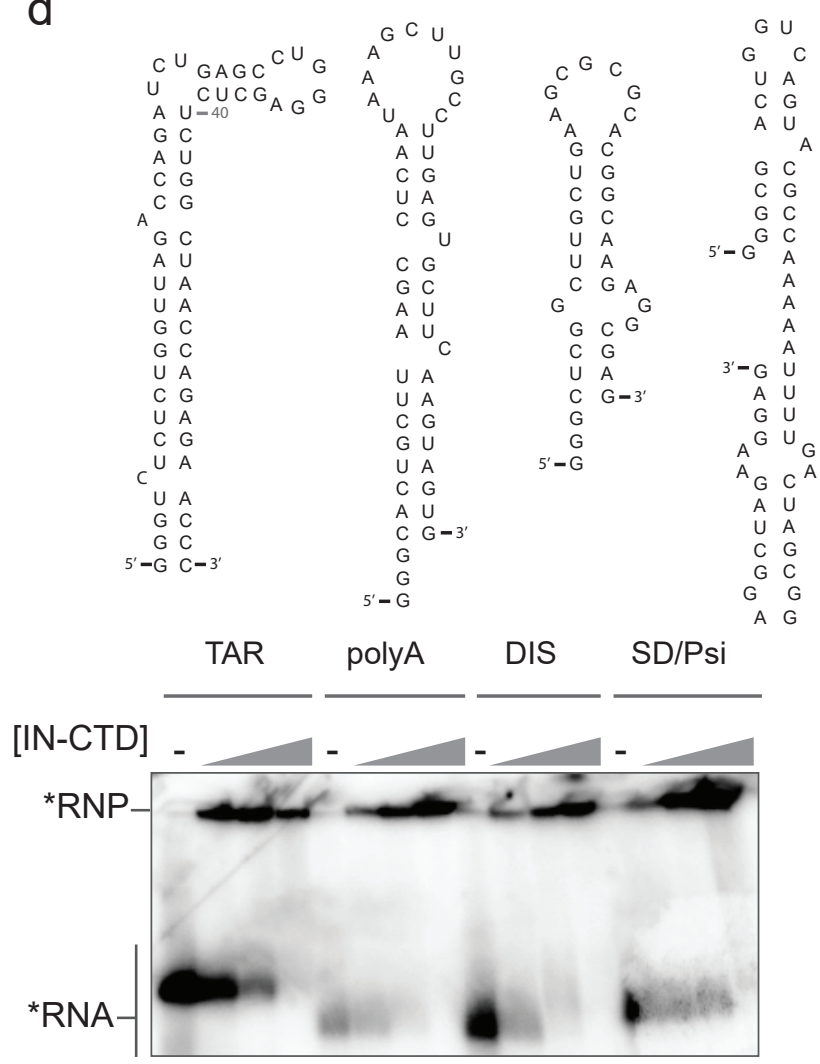

f
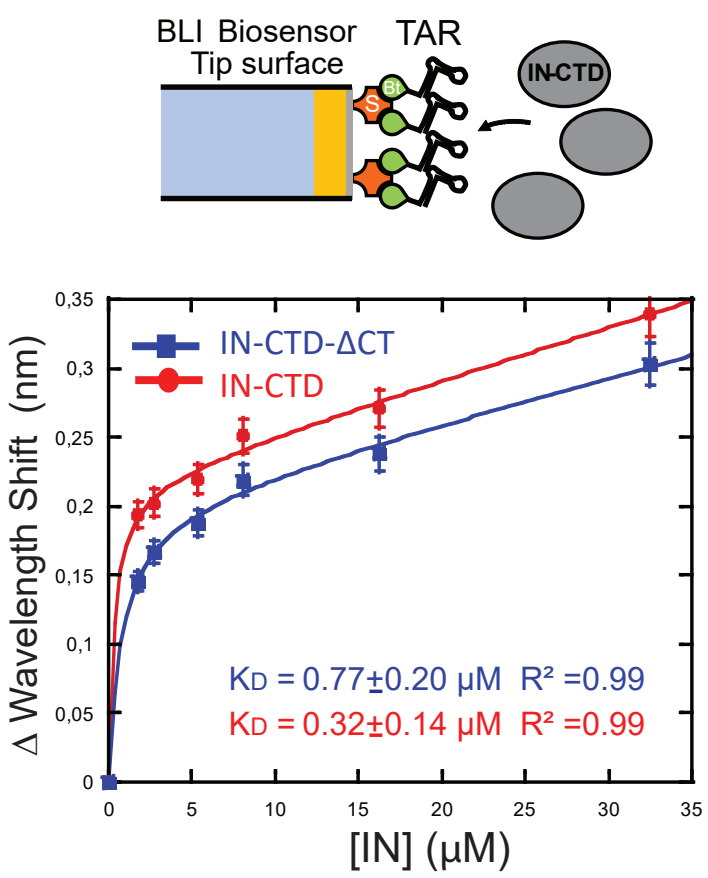
bioRxiv preprint doi: https://doi.org/10.1101/2021.10.21.465253; this version posted October 21, 2021. The copyright holder for this preprint (which was not certified by peer review) is the author/funder. All rights reserved. No reuse allowed without permission.

Figure 2

a

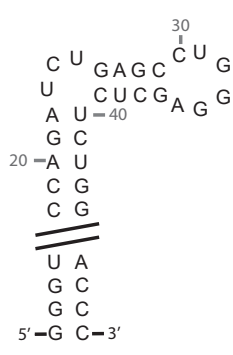

TAR

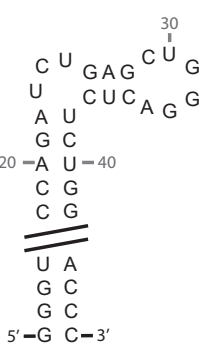

TAR-SS
C

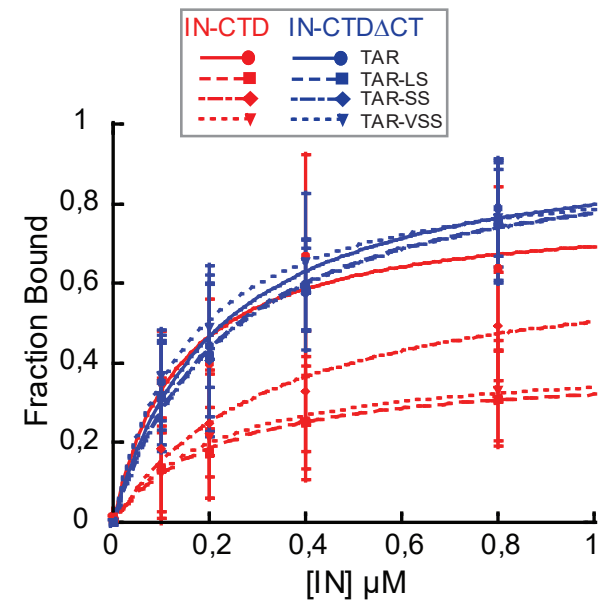

b

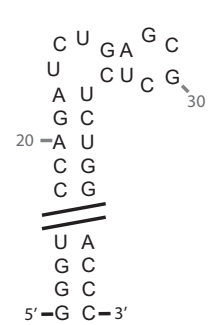

TAR-VSS

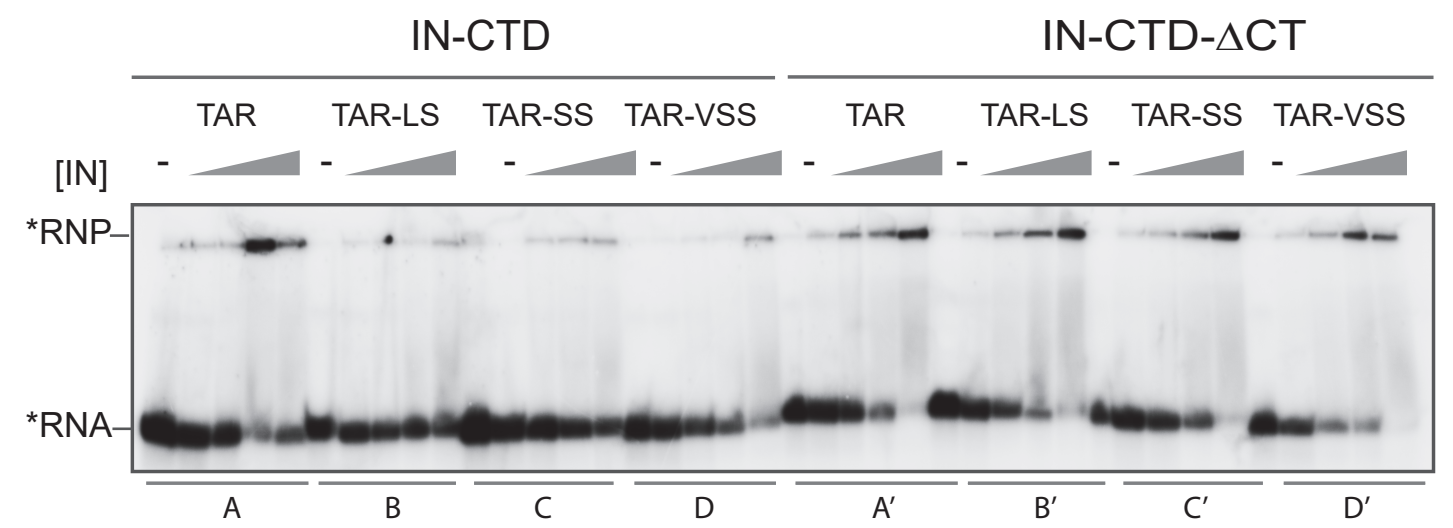


Figure 3

a
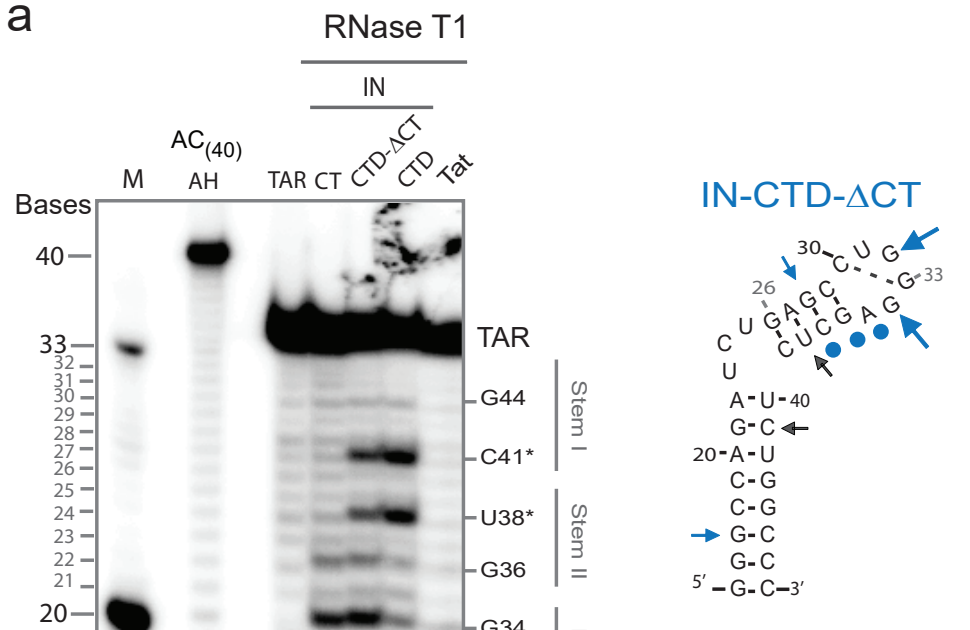

d

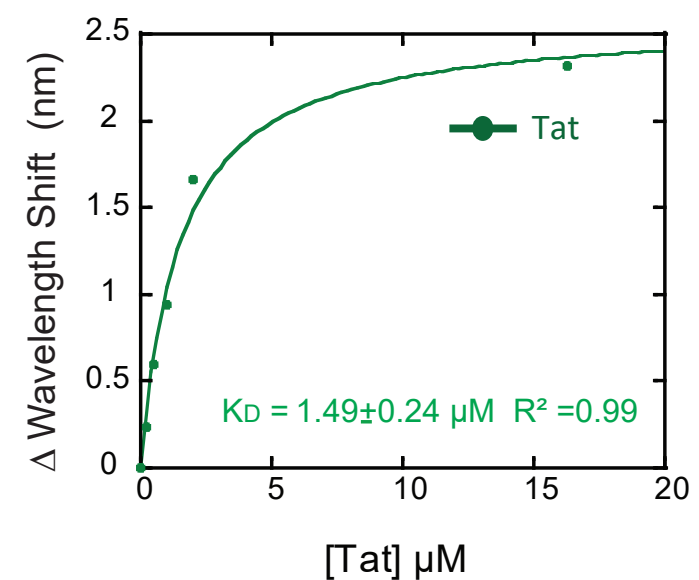

b

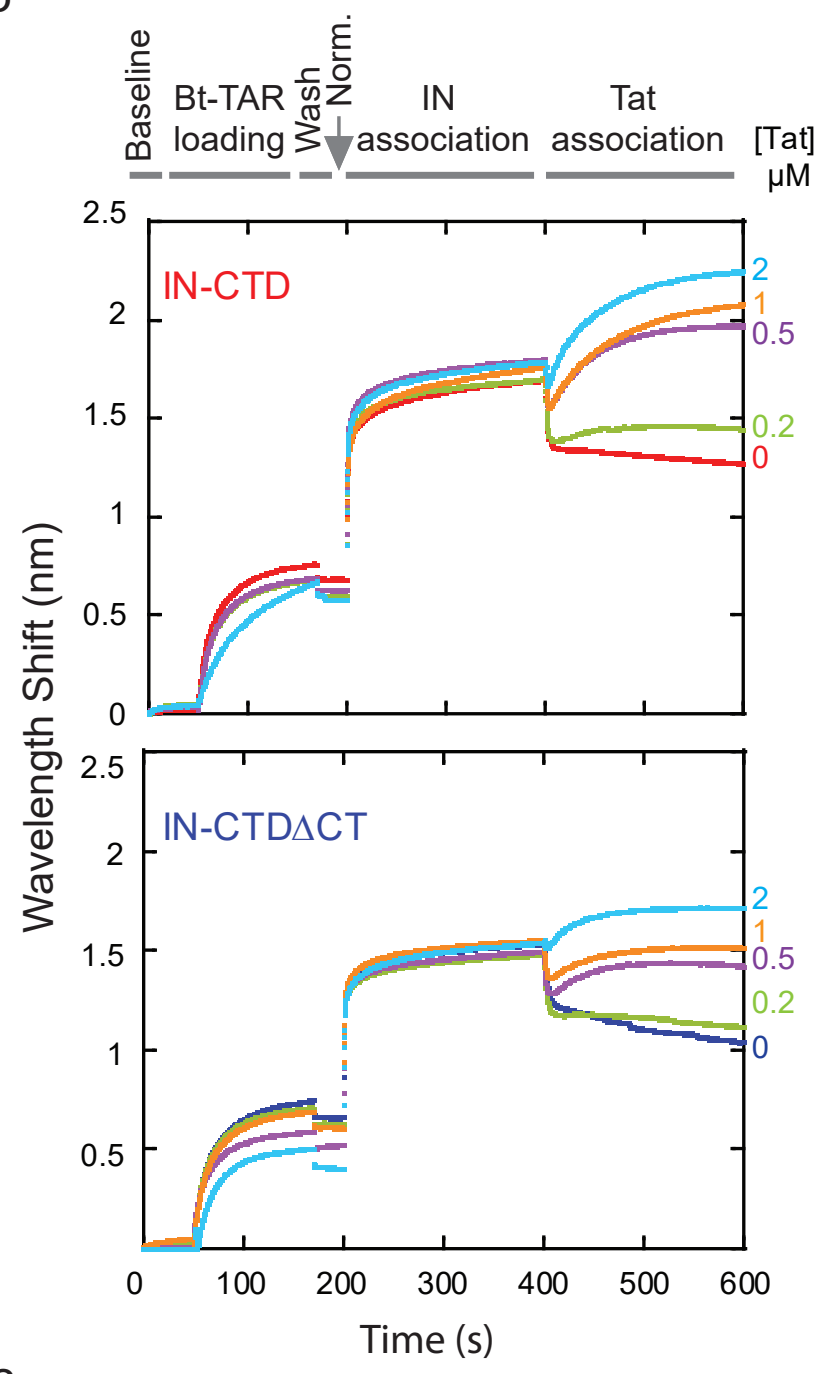

C

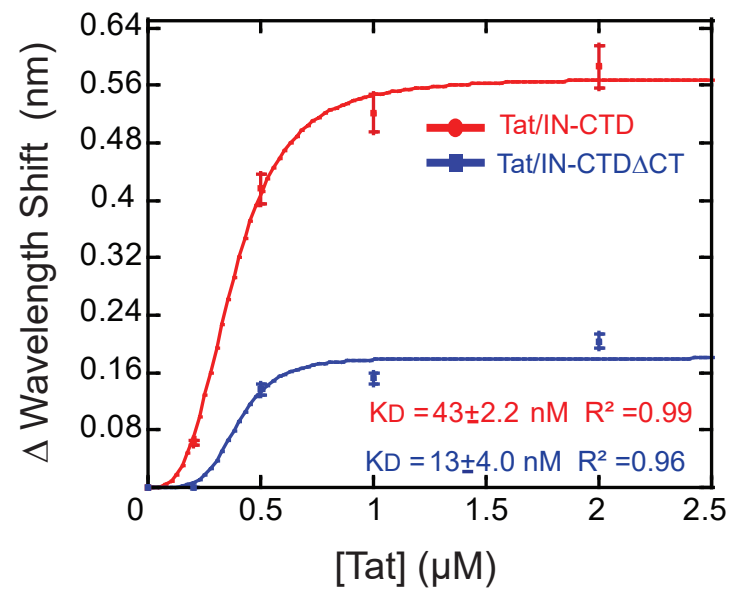


bioRxiv preprint doi: https://doi.org/10.1101/2021.10.21.465253; this version posted October 21, 2021. The copyright holder for this preprint (which was not certified by peer review) is the author/funder. All rights reserved. No reuse allowed without permission.

\section{Figure 4}

a

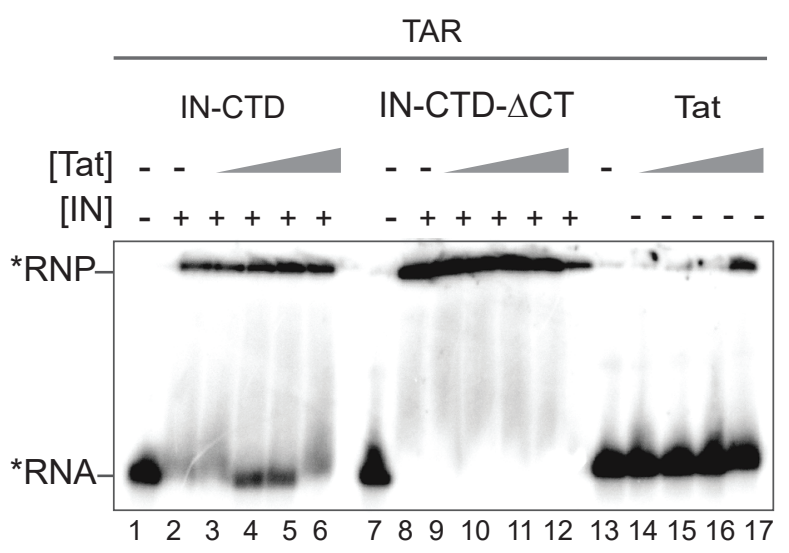

b

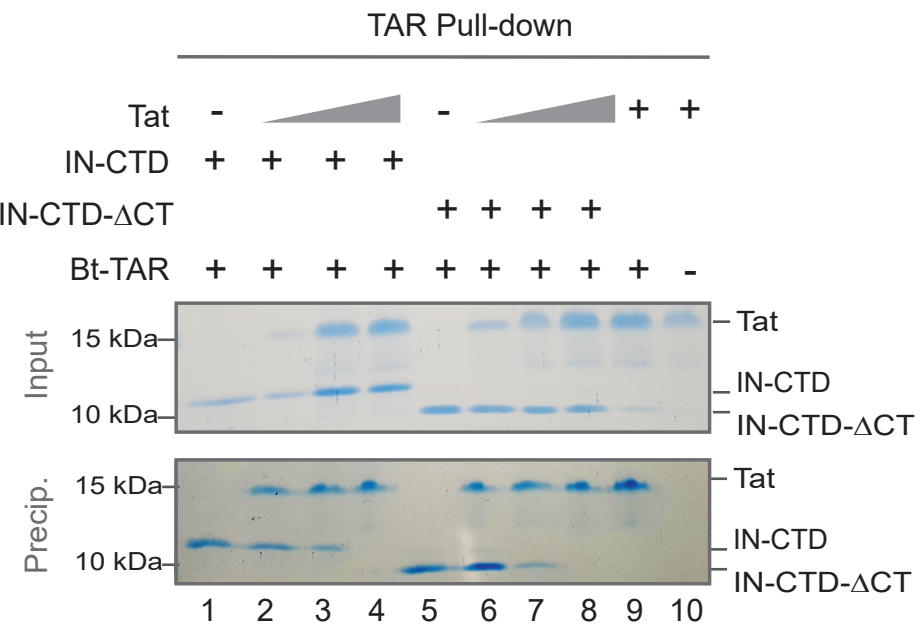




\section{Figure 5}
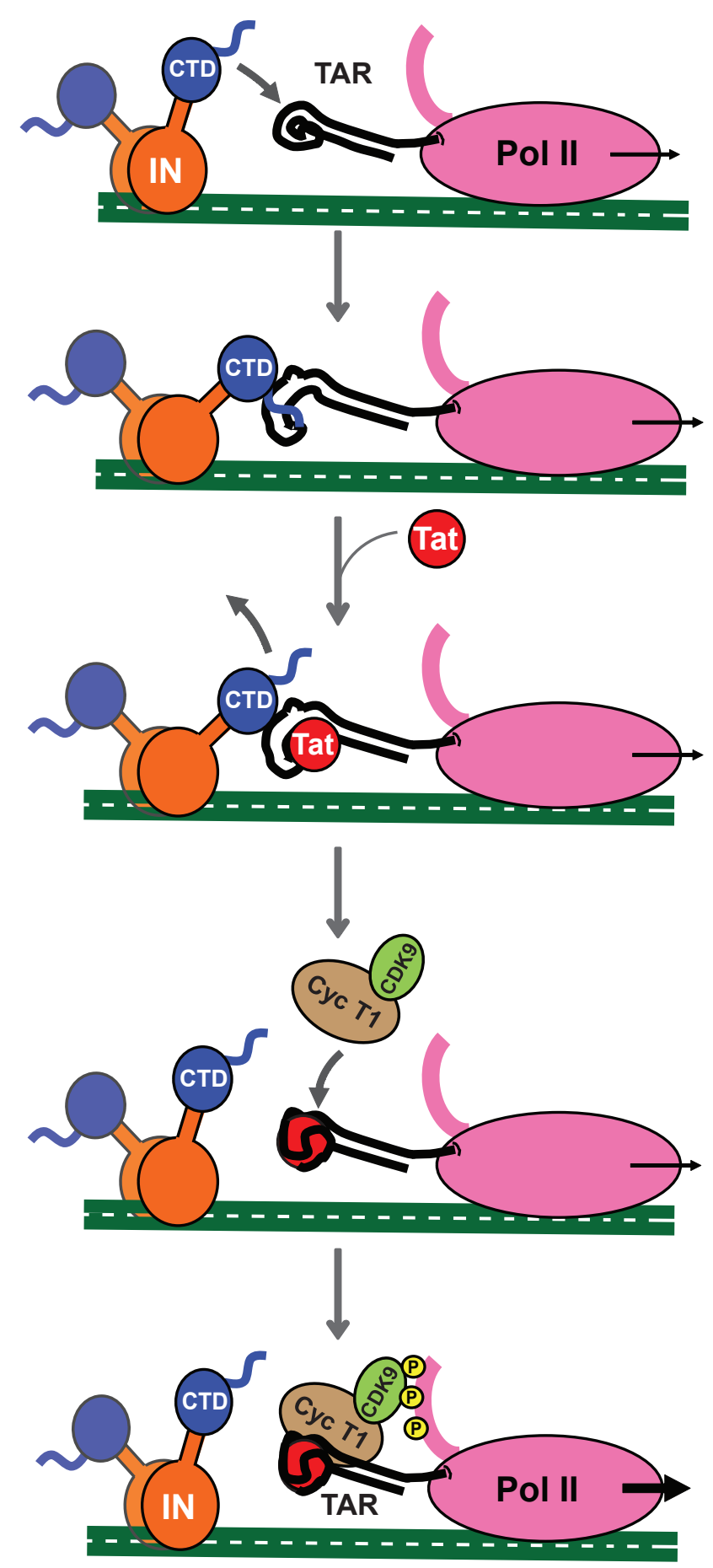


\begin{tabular}{|l|l|l|}
\hline Table 1 RNAs used in this study \\
\hline Name & Sequence (5'-3') & $\begin{array}{l}\text { Oligonucleotides and RNA } \\
\text { synthesis }\end{array}$ \\
\hline AG $_{(50)}$-mer & gggagagagagagagagagagagagagagagagagagagagagagagaga & FF48/FF54 hybridization \\
\hline RNA $_{(30)}$-mer & cguccaucuggucaucuagugauaucaucg & chemically synthesized \\
\hline TAR & gggucucucugguuagaccagaucugagccugggagcucucuggcuaacuagggaaccc & FF90/FF135 hybridization \\
\hline polyA & gggcacugcuuaagccucaauaaagcuugccuugagugcuucaaguagug & FF155/FF156 - PCR from pNL4-3 \\
\hline DIS & gggcucggcuugcugaagcgcgcacggcaagaggcgag & FF157/FF158 - PCR from pNL4-3 \\
\hline SD/4 & gggcgacuggucaguacgccaaaaauuuugacuagcggaggcuagaaggag & FF159/FF160 - PCR from pNL4-3 \\
\hline TAR-LS & gggucucucugguuagaccagaucugagcgcugggacgcucucuggcuaacuagggaac & FF91/FF136 hybridization \\
\hline TAR-SS & gggucucucugguuagaccagaucugagcugggacucucuggcuaacuagggaaccc & FF92/FF137 hybridization \\
\hline TAR-VSS & Gggucucucugguuagaccagaucugagcgcucucuggcuaacuagggaaccc & FF93/FF138 hybridization \\
\hline Bt-TAR & gggccagaucugagccugggagcucucuggccc & chemically synthesized \\
\hline AC $(40)$ & acacacacacacacacacacaaaaaaaaaaaaaaaaaaaa & chemically synthesized \\
\hline
\end{tabular}


Table 2 Oligonucleotides used to produce RNA substrates

\begin{tabular}{|c|c|}
\hline Name & Sequence (5'-3') \\
\hline FF48 & AAATAATACGACTCACTATAGGG \\
\hline FF54 & 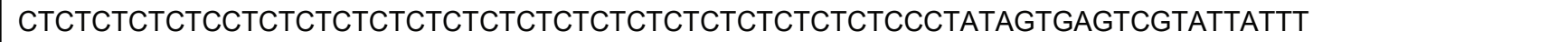 \\
\hline FF135 & AAATAATACGACTCACTATAGGGTCTCTCTGGTTAGACCAGATCTGAGCCTGGGAGCTCTCTGGCTAACTAGGGAACCC \\
\hline FF90 & GGGTTCCCTAGTTAGCCAGAGAGCTCCCAGGCTCAGATCTGGTCTAACCAGAGAGACCCTATAGTGAGTCGTATTATTT \\
\hline FF136 & AAATAATACGACTCACTATAGGGTCTCTCTGGTTAGACCAGATCTGAGCGCTGGGACGCTCTCTGGCTAACTAGGGAACCC \\
\hline FF91 & GGGTTCCCTAGTTAGCCAGAGAGCGTCCCAGCGCTCAGATCTGGTCTAACCAGAGAGACCCTATAGTGAGTCGTATTATTT \\
\hline FF137 & AAATAATACGACTCACTATAGGGTCTCTCTGGTTAGACCAGATCTGAGCTGGGACTCTCTGGCTAACTAGGGAACCC \\
\hline FF92 & GGGTTCCCTAGTTAGCCAGAGAGTCCCAGCTCAGATCTGGTCTAACCAGAGAGACCCTATAGTGAGTCGTATTATTT \\
\hline FF138 & AAATAATACGACTCACTATAGGGTCTCTCTGGTTAGACCAGATCTGAGCGCTCTCTGGCTAACTAGGGAACCC \\
\hline FF93 & GGGTTCCCTAGTTAGCCAGAGAGCGCTCAGATCTGGTCTAACCAGAGAGACCCTATAGTGAGTCGTATTATTT \\
\hline FF155 & AAATAATACGACTCACTATAGGGCACGCAAGCCCAAAAAAGCGCCGAGGCCAAAGAGG \\
\hline FF156 & CCTCTTTGGCCTCGGCGCTTTTTTGGGCTTGCGTGCCCTATAGTGAGTCGTATTATTT \\
\hline FF157 & AAATAATACGACTCACTATAGGGCTCGGCTTGCTGAAGCGCGCACGGCAAGAGGCGAG \\
\hline FF158 & CTCGCCTCTTGCCGTGCGCGCTTCAGCAAGCCGAGCCCTATAGTGAGTCGTATTATTT \\
\hline FF159 & AAATAATACGACTCACTATAGGGCGACTGGTGAGTACGCCAAAAATTTTGACTAGCGGAGGCTAG \\
\hline FF160 & CTAGCCTCCGCTAGTCAAAATTTTTGGCGTACTCACCAGTCGCCCTATAGTGAGTCGTATTATTT \\
\hline
\end{tabular}

